\title{
Periodismo colombiano en Twitter: usos de la herramienta en el ejercicio de la profesión
}

\section{Colombian journalism on Twitter: use of the tool in the professional practice}

\section{Jornalismo colombiano no Twitter: usos da ferramenta no exercício profissional}

Liliana Gutiérrez-Coba, Universidad de La Sabana, Bogotá, Colombia (liliana.gutierrez1@unisabana.edu.co)

Laura Silva-Gómez, Universidad de La Sabana, Bogotá, Colombia (laurasigo@ unisabana.edu.co)

RESUMEN | Esta investigación busca establecer cómo utilizan los periodistas colombianos Twitter para buscar información, construir mensajes y crear comunidades. En el estudio, 300 periodistas de 120 medios participaron en una encuesta, se realizaron 20 entrevistas a profesionales y un análisis de contenido de publicaciones en Twitter durante 2018 y 2019. Los resultados permiten establecer que, pese a las variadas posibilidades que ofrece esta plataforma para elaborar mensajes informativos innovadores y multimediales, la mayoría de los periodistas participantes en el estudio nolas utilizanibuscagenerar interactividad y comunicación bidireccional con sus audiencias.

PALABRAS CLAVE: Twitter; periodista; newsmaking; interactividad; Colombia. 
ABSTRACT/Based on a mixed methodology, which triangulates the data obtained in a survey of 300 journalists, 20 interviews and a content analysis, this research seeks to establish how Colombian journalists use Twitter to search for information, build messages, and create communities. The results allow us to establish that, despite the various possibilities that this platform allows for the construction of innovative and multimedia messages, most of the Colombian journalists surveyed do not use them nor do they seek to generate interactivity and two-way communication with their audiences.

KEYWORDS: Twitter; journalist; newsmaking; interactivity; Colombia.

RESUMO | Este artigo busca estabelecer como os jornalistas colombianos usam o Twitter para procurar informação, construir a mensagem e criar comunidades virtuais. No estudo participaram 300 jornalistas de 120 meios de comunicação em um inquérito, foram realizadas 20 entrevistas a profissionais e uma análise de conteúdo a publicações no Twitter durante 2018 e 2019. Os resultados obtidos revelam que, apesar de ter várias possibilidades na construção de mensagens inovadores e multimídia, a maioria dos jornalistas colombianos não estão usando nem gerando interatividade e comunicação bidirecional com seu público-alvo.

PALAVRAS-CHAVE: Twitter; jornalista; newsmaking; interatividade; Colômbia. 


\section{INTRODUCCIÓN}

El crecimiento de acceso a Internet en el mundo ha sido una constante. En enero de 2020 había 4540 millones de usuarios activos, 5190 millones de usuarios únicos de teléfonos móviles, y 3800 millones de usuarios de redes sociales (Kemp, 2020). Esta acelerada expansión ha traído cambios importantes en el periodismo, tanto en el ámbito del ejercicio profesional como en el funcionamiento de las empresas de comunicación, que han tenido que adaptarse a esta nueva realidad, y en especial a las redes sociales, herramientas indispensables para la labor diaria de los periodistas.

Las redes sociales son plataformas que han cambiado los comportamientos de quienes las consumen, generando nuevos hábitos en la labor periodística, siendo Twitter y Facebook las principales redes sociales que influyen en las prácticas e identidades profesionales, en relación con la verificación, la construcción de audiencias leales y la autorregulación de la profesión (Posetti, 2018). Tal como señala Hermida (2013), las redes sociales se han convertido en espacios donde las noticias se filtran, se debaten, se verifican de manera colaborativa, dando así a las audiencias un protagonismo antes impensable.

Adaptarse a este nuevo ecosistema implicó para los medios tradicionales, e incluso para los nativos digitales, la necesidad de aprender sobre el funcionamiento de las redes sociales y sus lenguajes, ya que solo entendiendo la lógica de cada red era posible lograr viralizar los contenidos. La distribución empezó a ser una función que debía cumplirse desde las redacciones, algo nuevo para los medios y sus periodistas, al mismo tiempo que las audiencias tienen más facilidades para leer noticias, colaborar, compartir y discutir la información (Gobbi, 2017; Lasorsa et al., 2012).

Asimismo, aspectos como el contacto con las fuentes y el reporteo se modificaron con la llegada de las redes sociales, ya que muchos periodistas comenzaron a usar la información presente en esta red como fuente primaria de sus productos (Cela et al., 2019; García-Borrego \& Campos, 2016). En síntesis, se produjo un cambio en las rutinas profesionales, entendidas como aquellos procedimientos habituales que se aceptan como apropiados por quienes ejercen un trabajo y que les permiten dilucidar, ante una situación típica, lo que se debe y lo que no se debe hacer (Lecaros \& Greene, 2012).

Todo ello ha generado en los periodistas la necesidad de establecer una eficaz conexión con la audiencia por medio de una actitud empática y colaborativa (Sánchez-Gonzales \& Méndez-Muros, 2015; López-Hermida \& Claro-Montes, 2011), conscientes de que las redes sociales son herramientas para obtener información, crear comunidades, atraer nuevas audiencias, y distribuir y viralizar contenidos (Carrera et al., 2012). 


\begin{tabular}{cc}
$\begin{array}{c}\text { Representaciones } \\
\text { gráficas }\end{array}$ & $\begin{array}{c}\text { Apoyo visual que muestra la actualidad informativa } \\
\text { de manera clara. Ejemplo: fotos y caricaturas. }\end{array}$ \\
\hline Infografías & Representar datos de manera gráfica y simbólica. \\
\hline Videos & Apoyo a la claridad, brevedad y eficacia narrativa. \\
\hline GIFs & Componente popular con toque humorístico. \\
\hline
\end{tabular}

Tabla 1. Formatos de la imagen en Twitter

Fuente: Elaboración propia con base en García-Avilés y Arias (2016).

Dentro del amplio espectro de redes sociales, Twitter es la herramienta más apropiada para desenvolverse en el trabajo periodístico, recopilar y reportar noticias (Hanusch \& Bruns, 2017; Powers \& Vera-Zambrano, 2018; Tandoc \& Vos, 2016), así como para mantener y afianzar las relaciones cercanas con la audiencia (Marta-Lazo \& García- Idiakez, 2014) o un recurso para comunicar novedades de manera sencilla (Crucianelli, 2010). La limitación de caracteres implica un cambio sustancial en la estructura narrativa periodística (Hermida, 2013) y la práctica de embeber tuits en las noticias se ha vuelto común para respaldar o ilustrar un argumento dado en el texto (Oschatz et al., 2021).

La Primera Gran Encuesta TIC (Ministerio de Tecnologías de la Información y Comunicaciones, 2017) destacó que $97 \%$ de la población colombiana que usa Internet lo hace para comunicarse y $79 \%$ tiene actividad en las redes sociales. Twitter es la quinta red social más usada (20\%) en el país, después de Facebook (88\%), WhatsApp (87\%), Instagram (34\%) y Google+ (29\%).

Diversos autores han estudiado desde diferentes aristas la manera en la que Twitter ha penetrado en las rutinas laborales de los periodistas, las motivaciones y usos de la plataforma, la exploración de narrativas y elementos interactivos para estrechar los vínculos de comunicación (Pérez-Soler \& Micó-Sanz, 2015; Burton \& Soboleva, 2011; Pentina et al., 2016).

De la variedad de elementos que Twitter permite para acompañar el mensaje, García-Avilés y Arias (2016) consideraron cuatro formatos visuales básicos para distribuir contenido en esta red: representaciones gráficas, infografías, videos y GIFs, como se ve en la tabla 1.

Por otra parte, las redes sociales han impulsado la interactividad como una forma de generar engagement con las audiencias, entendido como el estado psicológico de los usuarios que los acerca al emisor de los mensajes y que se manifiesta en acciones de diferente intensidad, como leer, comentar o aportar información nueva, 
dar me gusta o compartir una publicación (Bonsón et al., 2013; Barger \& Labrecque, 2013; Paine, 2011). El engagement implica, entonces, una interacción de doble vía entre quien comunica y quien recibe la información, y es altamente deseable, ya que las audiencias crean valor al contribuir a completar, recomendar o distribuir un contenido (Dolan et al., 2016; Ballesteros, 2019).

Twitter ha facilitado diferentes formas para que el periodista pueda involucrar a su audiencia y establecer puntos de contacto bidireccional con ella, acortando las fronteras geográficas y temporales, lo que para autores como Rost (2012), Ure (2017), Carrera y sus colegas (2012), Noguera (2013) y López-Meri (2016) es la esencia de esta red social. Todo esto, en un contex to en el que el que el modelo de comunicación de uno a muchos cambió a uno donde muchas personas pueden participar, incluso de modo conversacional entre sí (McQuail, 2013). Sin embargo, todavía es visible, en algunos casos, la distancia que se mantiene entre el periodista y su audiencia, un fenómeno anterior a Twitter, planteado tiempo atrás por McQuail y sus colegas (1997), que permanece en la actualidad por tres razones: la concentración física que mantiene marginadas a las zonas rurales de las ciudades principales, el uso unidireccional de las tecnologías y un bajo nivel de retroalimentación, y las distancias ideológicas y culturales entre el periodista y su audiencia.

De esta manera, McQuail y sus colegas (1997) aseguran que se pueden dar tres tipos de relación periodista-audiencia:

1. Dominante: el profesional de la información define su rol de manera respetuosa frente a su audiencia, cuyo principal objetivo es dominar la comunicación, imponer opiniones y propósitos a los receptores.

2. Autista: el periodista se enfoca en sus habilidades y destrezas, juicios profesionales de sus colegas y en el entorno de trabajo.

3. Equilibrio/cambio: existe un deseo por crear un vínculo comunicativo con la audiencia, hay respuestas e intercambio de ideas sobre temas particulares y una afinidad entre las formas de pensamiento entre el periodista y sus receptores.

Entendiendo la importancia de la interactividad en los medios digitales, es fundamental comprender hasta qué punto los periodistas interactúan con su público en Twitter, les interesa fortalecer el lazo comunicativo con quienes los siguen e involucrar su profesión con estos. Marta-Lazo y GarcíaIdiakez (2014) sugieren que existen dos tipos de perfiles de periodistas en Twitter: el periodista informante, quien entiende que su principal función en la labor informativa y comunicativa es difundir noticias, y un segundo rol como generador de opinión, que agrega valor añadido al profesional. 


\begin{tabular}{ccc} 
Aplauso & Conversación & Amplificación \\
\hline $\begin{array}{c}\text { Acciones que indican } \\
\text { acuerdo, interés o empatía } \\
\text { con la publicación, como } \\
\text { los me gusta. }\end{array}$ & $\begin{array}{c}\text { Respuestas directas o } \\
\text { indirectas al contenido } \\
\text { (comentarios, menciones } \\
\text { y respuestas). }\end{array}$ & $\begin{array}{c}\text { Acciones por las que se } \\
\text { comparte y extiende una } \\
\text { publicación a una audiencia más } \\
\text { extensa (retuits o citar tuits) }\end{array}$ \\
\hline
\end{tabular}

Tabla 2. Las tres características de la interactividad

Fuente: Elaboración propia con base en Kaushik (2011).

Además, no se puede pasar por alto que Twitter como red social tiene fines personales y de ocio (Rodríguez- Ruibal \& García-López, 2013) y, precisamente, ocupa el tercer tipo de uso más empleado por los comunicadores, porque involucra varias facetas del ser humano, contribuye en la configuración de una identidad social y deja a un lado el otro para fortalecer el nosotros.

En el contexto de las formas de engagement o grado de compromiso que los seguidores tienen con una cuenta, las informaciones publicadas por los periodistas son evaluadas por los usuarios de diversas maneras, como comentar, retuitear, o calificar, entre otras acciones (Díaz Soloaga, 2018).

Ure (2017) asegura que existe un efecto de valoración de los contenidos y de aceptación a la propuesta comunicativa por parte de los usuarios, que contiene una carga emocional y puede ser catalogado como rating de interactividad. En Twitter se manifiesta con menciones, respuestas, me gusta y retuits. Sin embargo, también reconoce que estas formas de interacción son de tipo unidireccional, en lugar de constituir un ámbito de encuentro conversacional. Por su parte, Kaushik (2011) caracteriza tres conductas de interacción de los usuarios con las publicaciones (tabla 2).

Además, Ure (2017) menciona dos tipos de engagement: el primero, la acción estratégica, enfocada en los intereses de quien publica y las reacciones esperadas de los usuarios y el segundo, más difícil de cumplir, de acción comunicativa. Este intenta crear ámbitos de colaboración, en los que puede participar con sus propios recursos, ideas, experiencias y conocimientos.

Ante este panorama, surgen las siguientes cuestiones de investigación aplicadas al contexto del periodismo en Colombia:

P1. ¿Cómo utilizan Twitter los periodistas para conseguir información?

P2. ¿En qué medida los periodistas utilizan las posibilidades que proporciona Twitter al elaborar información para esta plataforma?

P3. ¿Cuáles son las formas de interacción entre los periodistas y sus seguidores, producto de los tuits publicados a través de sus cuentas de Twitter? 


\section{MÉTODO}

Se empleó un método mixto, basado en técnicas cuantitativas y cualitativas.

En primera instancia, se elaboró una encuesta en formato escala de Likert, donde 1 era nunca y 5 era siempre. La encuesta estaba constituida por cinco ítems sobre el uso de Twitter para conseguir información (Alfa de Cronbach=0,890), 15 ítems que indagaban por las prácticas de newsmaking (Alfa de Cronbach=0,919), y 11 ítems sobre las formas de interacción del periodista con la audiencia (Alfa de Cronbach=0,902).

Para seleccionar la muestra se consideró el mapa de medios elaborado por el Consejo de Redacción \& Poderopedia (http://poderopedia.org/mapademedios/ index/), que da cuenta de la existencia de 220 medios en el país, pues en Colombia no existe un censo confiable sobre el número de periodistas. La encuesta fue enviada por correo electrónico y Twitter a 500 periodistas y se logró la respuesta de 300 que trabajan en 123 medios de 24 ciudades, en el periodo comprendido entre febrero de 2018 y febrero de 2019.

La segunda técnica utilizada fue el análisis de contenido de los perfiles de 20 profesionales considerados influyentes, para obtener una radiografía de sus prácticas en el uso de Twitter como herramienta de su ejercicio profesional. Los criterios de selección fueron: uso diario de la plataforma, el propio periodista, y no un equipo digital, maneja su cuenta, y que la cuenta sea pública (no necesariamente verificada).

El criterio de influencia de la muestra lo determinó el índice de medición SparkScore, que mide el impacto de una cuenta de acuerdo con la media de retuits, me gusta, número de seguidores, listas en las que está y el compromiso de interacción. El rango de los periodistas escogidos osciló entre 30 y 75 sobre 100, criterio que cumplieron 20 profesionales durante el período en estudio.

El soporte de este instrumento fue un libro de códigos con siete variables sociodemográficas, 22 variables relacionadas con el mensaje publicado y los recursos empleados en la construcción del mensaje, y nueve variables sobre interactividad. Como unidad de análisis se tomaron los dos tuits diarios con más impacto de cada periodista, para un total de 1200 mensuales, durante 30 días, en septiembre de 2018. Algunos periodistas no trinaban más de una vez al día. Finalmente, se analizaron 1008 tuits de manera manual.

Además, se realizaron entrevistas a 20 periodistas en ejercicio, vinculados con medios de comunicación públicos y privados de ocho ciudades colombianas, con el objetivo de conocer la experiencia de diferentes profesionales, revelando trazos y tendencias en sus rutinas profesionales. Las conversaciones fueron grabadas en audio y, posteriormente, se transcribieron para su análisis. Cada periodista firmó un consentimiento informado sobre el estudio y aprobó su participación, aunque algunos pidieron mantener su nombre en reserva. 


\section{RESULTADOS}

La mayor parte de quienes contestaron la encuesta se ubican en el rango de edad entre los 26 y 30 años (22,3\%) y entre los 31 y 35 años (19,3\%). Le siguen los que tienen entre 36 y 40 años (14,7\%) y los menores de 25 años (14\%). Se puede hablar de una población de periodistas jóvenes, que se esperaría estuviera más familiarizada con el manejo de Twitter. El 69\% de los periodistas que contestó la encuesta son hombres. El 58,3\% tiene solo grado universitario, el 13,7\% especialización y el 22\%, maestría.

\section{Twitter, efectivo para obtener información}

De los 300 periodistas participantes en la encuesta, 75,4\% emplea Twitter para investigar los contenidos periodísticos; además, 73,7\% aseguró que lo usa para identificar tendencias, temáticas coyunturales y noticias que marcan la agenda informativa de Colombia.

El 48,7\% afirmó que por medio de Twitter ha contactado fuentes ciudadanas, de entidades públicas, instituciones privadas y expertos para elaborar sus contenidos periodísticos. De hecho, hay una relación estadísticamente significativa, que muestra que $37,7 \%$ de quienes usan esta herramienta para contactar fuentes también lo hacen para identificar tendencias y temas que marcan la agenda informativa, que les dan ideas para su trabajo periodístico (Coeficiente de contingencia=0,759; $p=0,000$ ).

Un 54,4\% afirmó que para contactar fuentes en Twitter utiliza mensajes directos y $51,4 \%$ dijo haber accedido a los testimonios e información de las fuentes buscando en sus cuentas.

También cabe destacar que 39\% de los periodistas que han contactado a sus fuentes por mensajes directos o tuits ha recibido respuesta por este mismo medio, resultando ser un canal de comunicación válido, que acorta notablemente las barreras geográficas y temporales para el proceso productivo de la noticia y que se genera debido al monitoreo constante de personalidades o instituciones en las redes sociales.

En las entrevistas realizadas, los periodistas coincidieron en que redes sociales como Twitter son empleadas para recopilar información, pero aclararon que salir a la calle a buscar historias sigue siendo esencial. "Las redes sociales son una herramienta, pero no la única válida. Aunque hoy el periodismo sea más de oficina, me gusta el contacto con la comunidad y para mí es esencial salir a la calle a buscar historias" (entrevistado de Telepacífico).

En cuanto al tiempo a invertir, una entrevistada menciona que "las redes sociales nos han cambiado las formas de buscar la información, porque son herramientas que traspasan fronteras. En varias oportunidades he contactado a diferentes futbolistas por medio de Facebook y Twitter para los informes o avances periodísticos" (periodista, sección deportes Caracol Radio). 


\section{Mensajes poco multimediales en Twitter}

Sobre los elementos empleados dentro del tuit, se puede afirmar que las imágenes o galerías fotográficas son las más empleadas por los periodistas. En la encuesta, 134 periodistas (44,7\%) aseguraron que siempre (18\%) y casi siempre $(26,7 \%)$ suelen adjuntar una fotografía al mensaje publicado; $28 \%$ dice hacerlo a veces, casi nunca el $13 \%$ y nunca el $14,3 \%$. Por su parte, en el análisis de contenido hubo $234(23,8 \%)$ tuits que incluían al menos una imagen de las cuatro posibles.

"Utilizo muchas fotos, sobre todo gráficas, tablas completas relacionadas con el balompié colombiano. Hay gente que me sigue solo por eso y aprovecho para mostrar mejor la información, porque sé que normalmente las muestran de manera superficial" (periodista, diario El Tiempo).

Otro periodista considera que "Twitter ha facilitado la búsqueda de imágenes para los diferentes contenidos periodísticos, esto hace que no haya excusa para sacar una nota con imágenes de baja calidad" (periodista, Telecafé).

El segundo recurso más utilizado fue el video. En esta categoría se consideraron los videos sin edición, los editados (con marcas de agua, cortinillas o banners, entre otros detalles) y los que son estilo Playground (aquellos con textos breves y precisos). En la encuesta, 61 personas dijeron que a veces $(20,3 \%$ ) y en la misma proporción casi nunca $(20,3 \%)$, publicaban videos, frente a un $36,3 \%$ que nunca lo hacía, $14,7 \%$ que casi siempre los publicaba y $8,3 \%$ que siempre publicaba un video. En el análisis de contenido, apenas 3,4\% de los tuits revisados subieron un video.

Hay quienes aprovechan efectos en la comunicación visual: "Me encanta subir los tuits con fotos y retocarlas con un filtro especial para darle protagonismo a la imagen. También suelo dar retuit a los mensajes que incluyen videos" (periodista, ESPN y RCN Radio).

El siguiente recurso más empleado fue el GIF. En la observación de los tuits publicados por los periodistas influyentes, solo seis de 1008 mensajes analizados tenía una animación gráfica, ninguna basada en periodismo de datos o tipo infografía. En la encuesta, 36,7\% de los participantes afirmó que nunca los usaba y $23,3 \%$ contestó que casi nunca.

Los componentes menos utilizados en la muestra observada fueron las encuestas, ya que solo cuatro periodistas $(0,4 \%)$ usaron este instrumento para hacer sondeos de opinión para sus seguidores y únicamente en una oportunidad una periodista transmitió a través de Periscope (0,01\%).

Respecto de otros componentes del tuit, 156 periodistas manifestaron en la encuesta que nunca $(26,3 \%)$ y casi nunca $(20,3 \%)$ suelen hacer hilos para conectar 
varios tuits relacionados. El 22\% decía hacerlos a veces, casi siempre el 19,3\% y siempre el 12\%. En el análisis de contenido tan solo 3,2\% (32 tuits) de los mensajes observados tenían esta característica que permite unir mensajes.

Dentro de los factores evaluados estaba el uso de hashtags y etiquetas de cuentas directamente implicadas en sus mensajes publicados. Los encuestados respondieron que casi siempre $(29,3 \%)$ y siempre (39\%) usaban un numeral para contextualizar y agrupar conversaciones sobre un tema determinado, y $63 \%$ etiqueta a las personas o instituciones públicas y privadas que servían de fuentes. No obstante, en el análisis de los tuits se ve que 15,4\% (155 tuits de 1008) incluía numeral (\#) para mensajes personales, periodísticos, promocionales y de opinión.

Sobre el mensaje, en los resultados de la encuesta se puede apreciar que 61,7\% de los participantes prefiere citar frases puntuales de sus fuentes entrevistadas.

Dentro de la arquitectura del tuit, además, se examinaron otros factores del mensaje relacionados con el objetivo comunicativo a transmitir: el uso de hipervínculos, de emojis y el tema desarrollado. De los 1008 trinos observados, 129 incluían al menos un emoticono, mientras que 87,1\% (878) no los empleó. Es importante mencionar que 104 mensajes fueron publicados por periodistas que trabajan en las secciones denominadas softnews, sobre todo para publicaciones de carácter personal, opinión y de tipo informativo, y quienes menos los emplearon trabajan en secciones hardnews (solo 23 mensajes).

Otro componente que se consideró fue si los periodistas ponían un hipervínculo en sus mensajes. Solo 17\% (171 tuits) incluía un enlace. El 87,71\% (coeficiente de contingencia $=0,301 ; \mathrm{p}=0,000$ ) de los periodistas que incluyeron un hipervínculo trabaja en las secciones hardnews. Aunque los hallazgos muestran una baja inclusión de hipervínculos en los mensajes (solo 171 de 1008), 91,8\% corresponde a noticias publicadas por medios de comunicación colombianos e internacionales, en las que el principal fin es atraer tráfico a los sitios web de las casas periodísticas en las que laboran. El 8,2\% restante redireccionaban hacia YouTube, la Fundación Gabriel García Márquez, la UEFA, Fayerwayer (sitio de tecnología), FLIP (Liga contra el silencio), Spotify y Shazam.

Los periodistas que laboran en las secciones hardnews (10 entrevistados) emplean Twitter principalmente para opinar (23,01\%), contar aspectos personales $(13,39 \%)$ e informar $(13,19 \%)$, mientras que los que trabajan en secciones soft suelen utilizarlo principalmente para informar $(11,11 \%)$, opinar $(10,61 \%)$ y para contar aspectos personales $(8,24 \%)$ (coeficiente de contingencia $=0,356 ; p=0,000)$. En la tabla 3 se observan los objetivos comunicativos de los periodistas en sus tuits. 


\begin{tabular}{ccc} 
Objetivo comunicativo & $\begin{array}{c}\text { Periodistas que trabajan en } \\
\text { secciones hardnews }\end{array}$ & $\begin{array}{c}\text { Periodistas que trabajan en } \\
\text { secciones softnews }\end{array}$ \\
\hline Informar & $13,19 \%$ & $11,11 \%$ \\
\hline Describir & $0,00 \%$ & $0,69 \%$ \\
\hline Preguntar & $1,78 \%$ & $1,38 \%$ \\
\hline Debatir & $0,79 \%$ & $0,25 \%$ \\
\hline Aclarar & $1,38 \%$ & $2,48 \%$ \\
\hline Analizar & $0,19 \%$ & $0,39 \%$ \\
\hline Opinar & $23,01 \%$ & $10,61 \%$ \\
\hline Promocionar & $8,23 \%$ & $2,58 \%$ \\
\hline Contar aspectos personales & $13,39 \%$ & $8,24 \%$ \\
\hline No se puede determinar & $0,09 \%$ & $0,22 \%$ \\
\hline Total & $62,05 \%$ & 37,95 \\
\hline
\end{tabular}

Tabla 3. Objetivos comunicativos de los periodistas según la sección en la que laboran Fuente: Elaboración propia.

El 27,8\% de los mensajes analizados es de carácter periodístico (en su mayoría sobre noticias deportivas), 26,09\% está vinculado con aspectos personales de los periodistas, $0,59 \%$ trata sobre un tema institucional, $10,21 \%$ son promocionales (en su mayoría sobre el medio de comunicación en el que laboran), 35,21\% fueron opiniones sobre política, deportes y aspectos sociales principalmente, y en 0,79\% no se pudo determinar la tipología. Asimismo, el cruce entre tipo de mensaje y objetivo comunicativo del tuit (coeficiente de contingencia= 0,$846 ; p=0,000$ ) mostró que $86,44 \%$ de los mensajes de tipo periodístico se publicó con el objetivo de informar, por encima de describir, aclarar o analizar.

\section{Twitter: ¿plataforma de interactividad unidireccional o bidireccional?}

El cruce de la variable de contribución de Twitter en la investigación del periodista también evidencia que en $46,33 \%$ de los casos (coeficiente de contingencia= 0,352; $\mathrm{p}=0,000$ ) nunca y casi nunca solicitan la colaboración de los seguidores de Twitter para que envíen videos, fotografías u otro material para la construcción de sus contenidos periodísticos.

Respecto de los resultados del análisis de contenido de los tuits, es pertinente iniciar con las generalidades. De las tres formas más populares de interactividad que ofrece Twitter (respuesta, retuit y me gusta), menos de 36\% de los periodistas a quienes se les revisó el perfil suele emplearlas continuamente. La acción más 
frecuente fue dar una respuesta directa en otro tuit, (35,4\%, 357 de 1008 tuits). La segunda actividad empleada, (19,1\%, 193 de 1008 tuits), fue dar me gusta a los comentarios de sus seguidores en sus tuits. En 15,1\% de los casos (152 de 1008 tuits), los profesionales dieron retuit con comentario y la participación más baja se dio a través de los retuits (rt), con 0,7\% de la totalidad de mensajes recopilados.

En esta investigación se puede apreciar que el tuit del análisis de contenido con más me gusta y retuits fue el que publicó la periodista de la Revista Semana María Jimena Duzán, con un aproximado de 17.200 me gusta y 5300 retuits, en el cual agradeció el apoyo por la tutela que le colocó el juez Daniel Hernández dos días antes por la columna de opinión que publicó en el medio en el que labora y en el que manifiesta su deseo por la libertad de prensa en Colombia. Por su parte, el mensaje que registró más respuestas fue el de la periodista Yolanda Ruiz de RCN Radio, con más de 1200, quien preguntó la opinión de las personas sobre el ingreso de animales a los restaurantes.

Los periodistas suelen responder, dar retuit y me gusta a los mensajes relacionados con contenido periodístico-informativo $(27,08 \%)$ e identificarse más con aquellos que contienen opinión, un punto de vista o un juicio sobre un suceso $(35,21 \%)$ (coeficiente de contingencia $=0,763 ; p=0,001)$. (tabla 4 ).

Los mensajes en los cuales los periodistas opinan sobre un tema son también aquellos en los que más suelen generar conversación con sus seguidores, de manera que se establece la relación bidireccional de comunicación que se esperaría del uso de Twitter.

Bajo esta misma tendencia, los periodistas no fomentan un hashtag (\#) para crear comunidades, agrupar conversaciones ni lo tienen como prioridad en el tuit con el objetivo de monitorear conversaciones con otras personas sobre temas específicos, puesto que 84,6\% (853) de los mensajes analizados no contenían ningún numeral.

Igualmente, se evidenció que solo cuatro mensajes contenían el formato de encuesta de los 1008 tuits; tres de ellos fueron diseñados por periodistas que laboran en medios radiales y el otro, por uno que trabaja en televisión, reflejando que el uso de encuestas no es una práctica frecuente para conocer la opinión de las personas en Twitter. Tampoco es un hábito de los periodistas analizados solicitar fotos, videos y demás recursos a sus seguidores, ya que 99,9\% no hacía este pedido, solo se registró un trino con este tipo de petición.

En este proceso de establecer una comunicación con otras personas y de conocer en qué tipo de publicaciones existe la intención de interactuar, se incluyó un cruce relacionado con la variable objetivo comunicativo, en el que se tuvieron en cuenta diferentes propósitos que tienen los periodistas al momento de publicar un mensaje, entre ellos: informar, describir, analizar, preguntar, debatir, aclarar, opinar, promocionar y contar aspectos de carácter personal. 
Tipo de mensaje

\begin{tabular}{cc}
\hline Periodístico & $27,08 \%$ \\
\hline Personal & $26,09 \%$ \\
\hline Institucional & $0,6 \%$ \\
\hline Promocional & $10,22 \%$ \\
\hline Opinión & $35,21 \%$ \\
\hline No se puede determinar & $0,8 \%$ \\
\hline
\end{tabular}

Tabla 4. Interactividad de los periodistas según el tipo de mensaje en Twitter

Fuente: Elaboración propia.

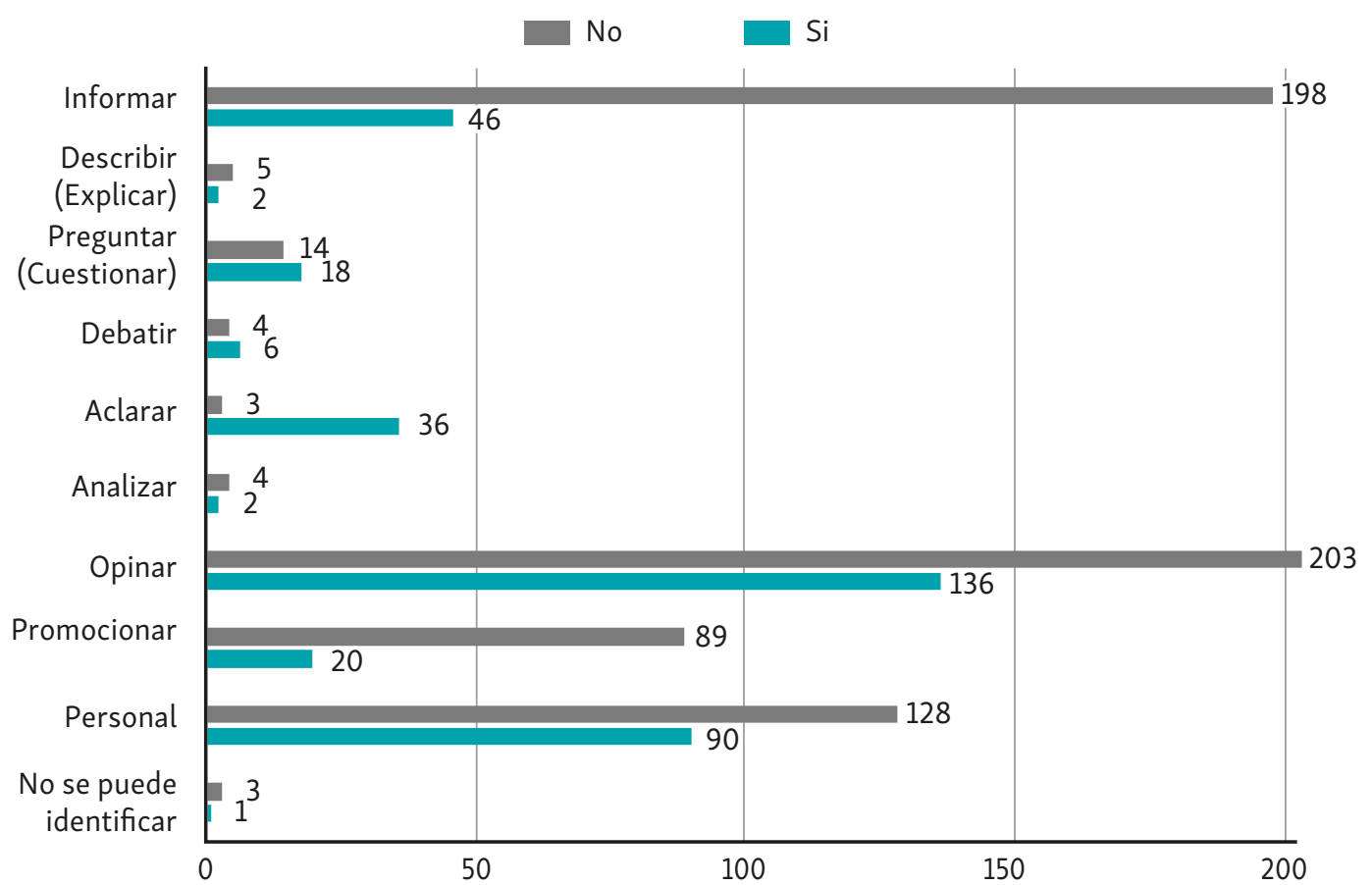

Figura 1. Respuesta de los periodistas a sus seguidores, según objetivo comunicativo del tuit

Fuente: Elaboración propia.

De los 339 tuits que fueron categorizados como opinión, los periodistas respondieron a 136 (40\%) intervenciones de sus seguidores. De estos 136 casos, $41 \%$ de las respuestas fueron dadas a mensajes que estaban relacionados con la vida personal de los periodistas (afinidades, experiencias, motivaciones, agradecimientos, etc.); asimismo, cuando publicaron mensajes de interés periodístico-informativo, para aclarar, cuestionar y analizar (coeficiente de contingencia $=0,319 ; p=0,000$ ), tal como se observa en la figura 1. 
Durante las entrevistas, los periodistas aseguraron estar pendientes de los comentarios que reciben y manifestaron su gusto por entablar conversaciones con sus seguidores. Por ejemplo, un comunicador de Canal Tro dijo que "cuando uno integra lo convencional con las nuevas tecnologías, logra tener una interacción directa con el televidente. Esas herramientas le hacen mucho bien al periodismo colombiano".

En el caso de El Colombiano, la editora general contó que, aunque ella no tiene cuenta en Twitter porque no le gusta la polémica y se siente expuesta por su oficio, en el periódico tienen diferentes espacios en redes sociales para interactuar con la gente y específicamente en Twitter está la Twittercrónica, una sección de noticias y crónicas compartidas en vivo con los seguidores de Twitter, en la que se hace "un ejercicio de reportería en tiempo real" sobre temas de la redacción (Medellín, moda, estilo de vida, redes sociales, entre otros). En el caso del Diario El Otún, el periodista contó que en la redacción suelen responder de vez en cuando a los comentarios, sobre todo para aclararle alguna situación a los usuarios, mientras que el profesional del diario La Libertad, de Barranquilla, aseguró que tiene en cuenta los comentarios, buenos y malos, porque todo sirve para mejorar y que interactúa constantemente en redes sociales porque recibe denuncias de la comunidad para cubrir en el medio.

En el análisis de contenido se pudo apreciar que los procesos de colaboración de los usuarios en los contenidos periodísticos son casi nulos. De los 1008 tuits analizados, la periodista Vanessa De La Torre, de Blu Radio, fue la única que solicitó a un seguidor evidencias sobre el tema de la tala de árboles que estaba desarrollando en su programa y que, posteriormente, publicó en otro tuit. De La Torre recibió un mensaje de un seguidor, quien le manifestó que tenía una carta en la que el Jardín Botánico aseguraba que mediante el Plan T replantaría los árboles en diferentes sectores de la ciudad. Ella le pidió una foto de tal prueba.

También se pueden apreciar dos tipos de relaciones opuestas entre el periodista y su audiencia propuestas por McQuail y sus colegas (1997): en primer lugar, una clara relación dominante por parte del periodista, quien es el sujeto que controla la comunicación, propone temáticas, impone opiniones, propósitos a los receptores y emplea Twitter como un soporte alternativo de producción y distribución de contenidos, complementando así el ejercicio periodístico. Sin embargo, también hay algunos tuits en los que se evidencia una posición de equilibrio/cambio, en los que el periodista tiene el deseo de crear lazos comunicativos con la audiencia, de intercambiar pensamientos y de conocer la pluralidad de ideas sobre temáticas específicas y de interés para ambas partes (McQuail et al., 1997). 


\section{DISCUSIÓN Y CONCLUSIONES}

Los resultados obtenidos indican que los periodistas ven en Twitter una herramienta con gran potencial para investigar información sobre los contenidos periodísticos que están elaborando, así como para identificar tendencias, temáticas circunstanciales según la ubicación geográfica y las principales noticias del momento. De esta manera, Twitter cumple con una de sus promesas de servicio, facilitando el proceso de búsqueda y tendencias para los profesionales de la información.

Los hallazgos también confirman la noción que García-Borrego \& Campos (2016) presentan sobre la búsqueda de fuentes a través de las redes sociales. Para los investigadores, el uso de herramientas como Twitter y Facebook con fines laborales es una experiencia de relacionamiento renovada de los periodistas con sus fuentes mediante las plataformas digitales. La mayoría de los periodistas participantes contactan a fuentes con diferentes características (públicas, privadas, ciudadanas) a través de Twitter, sobre todo por mensaje directo, porque permite un diálogo privado, confiable e inmediato con la fuente, convirtiéndose en un canal válido para acceder al testimonio de un sujeto determinante en el proceso de construcción del contenido. Sin embargo, hay que considerar que no era el único método para obtener testimonios de sus fuentes, ni remplaza el trabajo de reporteo.

Aunque Pérez-Soler y Micó-Sanz (2015) plantean que el contacto de fuentes a través de Twitter es una práctica secundaria, así como la interacción con audiencias y el espacio de debate, y que el único contexto para conectar con las fuentes mediante las redes sociales es cuando ocurre un acontecimiento no planificado, en este trabajo se encontró que la búsqueda de fuentes es uno de los principales usos que los periodistas colombianos hacen de Twitter, ya que es un canal directo, rápido y sin intermediarios, en el que las fuentes se expresan y en el que los periodistas pueden hacer un monitoreo constante.

Los resultados de este trabajo coinciden con Rost (2012), que evidencia la carencia de una narrativa que aproveche las características de Twitter y la ausencia de creatividad al momento de elaborar el mensaje. Por ejemplo, en esta investigación se percibió que los periodistas emplean muy poco los emojis, no usan los hashtags para crear una comunidad virtual y, en el contenido diferencial que se puede adjuntar al mensaje en formatos de imagen, los profesionales le apuestan casi exclusivamente a la fotografía. Hay un mínimo uso de las transmisiones con Periscope para los eventos en tiempo real, mientras que los GIFs con periodismo de datos y las infografías son los elementos que definitivamente no se emplean en el ejercicio diario de la profesión en esta plataforma. 
Esta falta de diversidad en el tipo de recursos utilizados para elaborar los mensajes en Twitter puede ser causa de la falta de tiempo de los periodistas, ya que crear recursos diferenciales puede demandar más dedicación, aunque también puede deberse a una falta de capacitación para manejar estas herramientas.

Las fotografías y los videos (editados o no) son los recursos más empleados por los periodistas al momento de construir el mensaje en Twitter, dos de los tres recursos que coinciden con el punto de vista de Lasorsa y sus colegas (2012), pues para los investigadores, los formatos visuales y digitales han contribuido a la forma en la que el periodista ejerce su profesión. Si bien los autores también mencionan la categoría de audio, en este trabajo no se encontró un tuit y tampoco se cuestionó sobre este recurso, pues al terminar esta investigación no era una función primaria de Twitter y era necesario recurrir a aplicaciones como AudioBoo, Chirbit, Shoutomatic y Audiolip.

En esta investigación se pudo observar que los periodistas emplean Twitter con algún elemento visual con el objetivo de informar, compartir una experiencia o afinidad personal, promocionar algún producto, servicio o contenido, y para opinar. Esto encaja con lo manifestado por García-Avilés y Arias (2016), puesto que los autores destacan la noticia como el principal género informativo cuyo principal acompañamiento es la fotografía porque permite contextualizar, y presentar los datos de manera concisa y contundente. Los periodistas colombianos deben reforzar el uso de la imagen, porque, como lo manifiestan los autores, cada vez es más importante este elemento en la comunicación digital, obliga a estar en la búsqueda constante de nuevos formatos narrativos, sin perder de vista la calidad y el rigor que exige la profesión.

Los principales usos que los periodistas de esta muestra le dieron a Twitter están relacionados con la opinión y la información periodística. Se presenta un hecho contrario al caso del estudio de López-Hermida y Claro-Montes (2011), cuyos resultados arrojaron que los comunicadores chilenos empleaban Twitter para conectarse con sus seguidores, no para informar ni tener una estrecha relación con el medio de comunicación en el que laboran. En Colombia, diversos estudios que observaron el comportamiento de las cuentas de los medios de comunicación coinciden con que usan Twitter como difusor de contenidos informativos de diversa índole (Santiesteban \& Rodríguez, 2013; Cobos, 2015; Espinosa \& Rincón, 2013; Hernández, 2016).

Al momento de determinar las formas de interacción entre los periodistas y su audiencia a través de sus cuentas de Twitter, esta investigación evidencia las tres características de interacción que tiene en cuenta Kaushik (2011), pero no desde la acción del periodista sino desde la audiencia, que reacciona superlativamente con lo que el autor denomina aplauso, acción que indica acuerdo o empatía y 
que puede entenderse como los me gusta; también cumple con la categoría de conversación, porque hay respuestas directas e indirectas al contenido, y además hay amplificación de los mensajes de los periodistas a través de los retuits. Estas acciones pueden ser la representación de lo que Mariano Ure (2017) denomina rating de interactividad, que se representa de diferentes maneras e involucra una carga emocional, o también lo que McCay-Peet y Quan-Haase (2016) definen como una experiencia de usuario con tecnologías que permiten interactuar, crear y compartir en sus redes sociales, y que se agrupan por una afinidad ideológica.

Esto, además de ratificar que los periodistas de la muestra emplean Twitter principalmente para informar, opinar y compartir experiencias personales, también corrobora que este tipo de publicaciones son las que más generan interacción por parte de sus usuarios, correspondiendo con lo planteado por Carmen Díaz-Soloaga (2018) al asegurar que el compromiso adquirido por los seguidores es fruto de la sensibilidad eimpacto del mensaje publicado y, al mismo tiempo, manifiesta un efecto de aceptación y valoración que puede ser clasificado como rating de interactividad unidireccional porque no construye un diálogo entre las partes (Ure, 2017).

Ahora bien, Rost (2012), Noguera (2013) y López-Meri (2016) mencionan que la interactividad es la característica más importante que define a los medios digitales y al lenguaje web, porque amplía la interconexión, la participación y los entornos de inmersión. En la investigación se puede apreciar que los comunicadores no interactúan con sus seguidores con respuestas, me gusta o retuits, de manera que es difícil valorar Twitter como un entorno comunicativo bidireccional en el caso del periodismo colombiano.

Los resultados encontrados en esta investigación contradicen a aquellos autores que plantearon la importancia de crear comunidades virtuales con numerales, con el objetivo de incrementar el diálogo y la interacción directa y continua con los usuarios hasta fidelizarlos (Manfredi et al., 2015), porque en los hallazgos se percibe que los periodistas no tienen como fin crear campos de unión, relaciones o conversaciones a través de un determinado hashtag.

Estas experiencias refuerzan las líneas de pensamiento planteadas por McQuail y sus colegas (1997), pues pese a que la esencia de las redes sociales es fortalecer la bidireccionalidad en un entorno ideal con sujetos, dispositivos e interfaces de por medio, realmente existe una comunicación unidireccional entre el periodista y su audiencia porque la retroalimentación es baja, así como el flujo activo de participación; apoyando la misma idea, Arrabal y De-Aguilera (2016) manifiestan que, aunque Twitter es una red social que ha intentado reforzar el poder de la interactividad, se desaprovechan múltiples formas de comunicación interpersonal y se olvida que las acciones deben girar en torno al usuario. 
Asimismo, en las prácticas que se observaron, a los periodistas les gusta proponer temas o preguntas para debatir con otros usuarios, pero no hay espacios para la participación en la construcción de contenidos, ya que no fomentan que les envíen preguntas para hacerle a un entrevistado, tampoco piden fotos o videos cuando ocurre un suceso noticioso ni utilizan los hashtags para formar comunidades, predominando la comunicación unidireccional y no sacando provecho de la bidireccionalidad que ofrece la herramienta.

Con base en los resultados, el desafío más grande para los periodistas colombianos en Twitter es generar mayor compromiso con la audiencia mediante el aprovechamiento de todas las posibilidades que permite la plataforma, tanto para la construcción de mensajes como para la interacción con los usuarios.

Es importante señalar que los periodistas colombianos desaprovechan varias funciones que tiene Twitter para crear contenidos creativos e impulsar su discurso en la plataforma, lo cual puede pasar porque los medios han definido de manera superficial el uso de Twitter en sus manuales de redacción, dando recomendaciones y principios de implementación, pero no con conceptos y un desglose detallado de cómo sacarle el máximo provecho a la plataforma, algo que se debe atender con urgencia.

\section{FINANCIAMIENTO}

Investigación financiada por la convocatoria interna a proyectos de investigación COM 71206 de la Universidad de La Sabana, Bogotá, Colombia.

Agradecemos a los miembros del Semillero de Calidad Informativa: Juan David Botero, Nicolás Medellín, Sebastián Vallejo, Camilo Páez y Javier Saba y al auxiliar de investigación , magíster Daniel Tobón.

\section{REFERENCIAS}

Arrabal, G. \& De-Aguilera, M. (2016). Comunicar en 140 caracteres. Cómo usan Twitter los comunicadores en España (Communicating in 140 characters. how journalists in Spain use Twitter). Comunicar, 46, 9-17. https://doi.org/10.3916/C46-2016-01

Ballesteros, C. (2019). La representación digital del engagement: hacia una percepción del compromiso a través de acciones simbólicas (Digital representation of engagement: towards a perception of commitment through symbolic actions). Revista de Comunicación, 18(1), 215-233. https://doi.org/10.26441/RC18.1-2019-A11 
Barger, V. A. \& Labrecque, L. (2013). An Integrated Marketing Communications Perspective on Social Media Metrics. International Journal of Integrated Marketing Communications, 5(1), 64-76. https://papers.ssrn.com/sol3/papers.cfm?abstract_id=2280132

Bonsón, E., Royo, S., \& Ratkai, M. (2015). Citizens' engagement on local governments' Facebook sites. An empirical analysis: The impact of different media and content types in Western Europe. Government Information Quarterly, 32(1), 52-62. https://doi.org/10.1016/J.GIQ.2014.11.001

Burton, S. \& Soboleva, A. (2011). Interactive or reactive? Marketing with twitter. The Journal of Consumer Marketing, 28(7), 491-499. https://doi.org/10.1108/07363761111181473

Carrera, P., Sainz, C., Herrero, E., \& Limón, N. (2012). Journalism and Social Media: How Spanish Journalists Are Using Twitter. Estudios sobre el mensaje periodístico, 18(1), 31-53. https://doi.org/10.5209/rev_ESMP.2012.v18.n1.39353

Cela, J., Parras, A., \& Romero, L. (2019). Uso de las redes sociales en diplomacia, política y relaciones internacionales. Análisis de la información publicada en las versiones online de dos periódicos españoles: "El País" y "La Vanguardia" (Use of Social Media in Diplomacy, Politics and International Affairs. Analysis of the Information published in two online Spanish Newspapers: El País and La Vanguardia). Estudios Sobre El Mensaje Periodístico, 25(2), 711-726. https://doi.org/10.5209/esmp.64798

Cobos, T. (2015). Radiografía al comportamiento de la prensa colombiana en Twitter: Casos El Heraldo, El Universal y El Informador (Radiography behavior of the Colombian press on Twitter: El Heraldo, El Universal and El Informador study cases). Revista Encuentros, 13(1), 85-99. https://doi.org/10.15665/re.v13i1.351

Crucianelli, S. (2010). Herramientas digitales para periodistas (Digital tools for journalists). Retrieved from https://journalismcourses.org/wp-content/uploads/2020/08/DigitalTools-for-Journalists-esp.pdf

Díaz-Soloaga, C. (2018, February 27). Calcular el engagement en Twitter, qué ayuda y qué no a mejorarlo (Calculate Twitter engagement, what helps and what does not to improve it). Social Media Pymes. Retrieved from https://www.socialmediapymes.com/calcular-el-engagement-en-twitter/

Dolan, R., Conduit, J., Fahy, J., \& Goodman, S. (2015). Social media engagement behaviour: a uses and gratifications perspective. Journal of Strategic Marketing, 24(3-4), 261-277. https://doi.org/10.1080/0965254X.2015.1095222

Espinosa, A. \& Rincón, D. (2013). ¿Cuál es la estrategia que usan los dos medios de mayor circulación en Colombia a través de Twitter? (What is the strategy used by the two media with the largest circulation in Colombia through Twitter?) (Doctoral dissertation, Universidad de La Sabana, Colombia). Retrieved from http://hdl.handle.net/10818/8932

García-Avilés, J. \& Arias, F. (2016). Géneros periodísticos en los formatos visuales de Twitter: una propuesta de tipología (Journalism genres in Twitter's visual formats: a proposal for classification). Textual \& visual media, 9, 101-132. ttps://textualvisualmedia.com/index. php/txtvmedia/article/view/52

García-Borrego, M. \& Campos, S. (2016). Rutinas profesionales de los becarios de Periodismo: el caso de Málaga (Media routines of Journalism interns: the case of Málaga). ZER-Revista de Estudios de Comunicación=Komunikazio Ikasketen Aldizkaria, 21(41), 155-169.

https://doi.org/10.1387/zer.17267 
Gobbi, J. (2017). Información digital y nuevas formas de construcción de la agenda de noticias (Digital information and new ways of building the news agenda). Sociales en debate, 12, 2936. https://publicaciones.sociales.uba.ar/index.php/socialesendebate/article/view/3238

Hanusch, F. \& Bruns, A. (2017). Journalistic Branding on Twitter. A representative study of Australian journalists' profile descriptions. Digital Journalism, 5(1), 26-43. https://doi.org/10.1080/21670811.2016.1152161

Hermida, A. (2013). \#Journalism: Reconfiguring Journalism Research About Twitter, One Tweet at a Time. Digital Journalism 1(3), 295-313. https://doi.org/10.1080/21670811.2013.808456

Hernández, J. (2016). Interactividad y socialización periodística en Twitter. Un análisis de los medios migrantes colombianos de mayor influencia online (Journalistic Interactivity and Socialization in Twitter. An Analysis of Migrant Media with More Online Influence in Colombia). Estudios sobre el mensaje periodístico, 22(2), 1065-1083. https://doi. org/10.5209/ESMP.54252

Kaushik, A. (2011, October 10). Best Social Media Metrics: Conversation, Amplification, Applause, Economic Value. Occam's Razor. Retrieved from https://www.kaushik.net/avinash/best-social-media-metrics-conversation-amplification-applause-economic-value/

Kemp, S. (2020, January 30). Digital 2020: 3.8 Billion People Use Social Media. WeAre Social. Retrieved from https://wearesocial.com/blog/2020/01/digital-2020-3-8-billion-people-use-social-media

Lasorsa, D. L., Lewis, S. C., \& Holton, A. E. (2012). Normalizing Twitter. Journalism practice in an emerging communication space. Journalism Studies, 13(1), 19-36. https://doi.org/10.1080/1461670X.2011.571825

Lecaros, M. J. \& Greene, F. (2012). Editores chilenos y rutinas periodísticas: revisión y propuesta para un estudio sobre el cambio en la sala de redacción (Chilean Editors and Journalistic Routines: Review and Proposal Towards a Study on Change in Newsrooms). Cuadernos.info, 30, 53-60. https://doi.org/10.7764/cdi.30.426

López-Hermida, A. \& Claro-Montes, C. (2011). Medios y periodistas en Twitter: el caso chileno (Media and journalists on Twitter: the Chilean case). Correspondencias \& Análisis, 1, 17-33. https://doi.org/10.24265/cian.2011.n1.02

López-Meri, A. (2016). Periodismo en Twitter. La contribución de los usuarios al flujo informativo (Journalism on Twitter. The users' contribution to the flow of information). Cuadernos.info, 39, 241-257. https://doi.org/10.7764/cdi.39.825

Manfredi, J., Rojas, J. L., \& Herranz. J. (2015). Entrepreneurial journalism: Sports journalism in Spain. Revista Latina De Comunicación Social, 70, 69-90. https://doi.org/10.4185/RLCS-2015-1035

Marta-Lazo, C. \& García-Idiakez, M. (2014). El uso profesional de la red social Twitter en la redacción del diario español El País (Professional Use of the Social Networking Service Twitter in Composing the Spanish Newspaper El País). Palabra Clave, 17(2), 353-377. https://doi.org/10.5294/pacla.2014.17.2.4

McCay-Peet, L. \& Quan-Haase, A. (2016). A model of social media engagement: User profiles, gratifications, and experiences. In H. O’Brien \& M. Lalmas (Eds.), Why engagement matters: Cross-disciplinary perspectives and innovations on user engagement with digital media (pp. 199-217). Springer Verlag. 
McQuail, D. (2013). Reflections on paradigm change in communication theory and research. International Journal of Communication, 7(14), 217-229. https://ijoc.org/index.php/ijoc/article/viewFile/1961/850

McQuail, D., Windhal, S., López-Escobar, E., \& Sádaba, C. (1997). Modelos para el estudio de la comunicación colectiva (Models for the study of collective communication). Eunsa

Ministerio de Tecnologías de la Información y Comunicaciones. (2017). Primera Gran Encuesta TIC (First large ICTs survey). Colombia TIC. Retrieved from https://colombiatic. mintic.gov.co/679/w3-article-74002.html\#: :text=La\%20primera\%20Gran\%20 Encuesta\%20TIC,producidos\%20desde\%20el\%20Ministerio\%20TIC

Noguera, J. (2013). How open are journalists on Twitter? Trends towards the end-user journalism. Comunicación y Sociedad, 26(1), 93-114. https://hdl.handle.net/10171/35432

Oschatz, C., Stier, S., \& Maier, J. (2021). Twitter in the News: An Analysis of Embedded Tweets in Political News Coverage. Digital Journalism. https://doi.org/10.1080/21670811.2021.1912624

Paine, K. D. (2011). Measure What Matters: Online Tools for Understanding Customers, Social Media, Engagement, And Key Relationships. John Wiley and Sons.

Pentina, I., Basmanova, O., \& Zhang, L. (2016). A cross-national study of twitter users' motivations and continuance intentions. Journal of Marketing Communications, 22(1), 36-55. https://doi.org/10.1080/13527266.2013.841273

Pérez-Soler, S. \& Micó-Sanz, J. (2015). El mito de la conversación global. Usos de Twitter en las redacciones periodísticas catalanas y belgas (The myth of the global conversation. uses of Twitter in Catalan and Belgian newsrooms). El profesional de la información, 24(3), 246-255. https://doi.org/10.3145/epi.2015.may.04

Posetti, J. (2018). News Industry Transformation: Digital Technology, Social Platforms and the Spread of Misinformation and Disinformation. In C. Ireton \& J. Posetti (Eds.). Journalism, 'fake news' and disinformation: A handbook for journalism education and training. Unesco. https://bit.ly/2XLRRlA

Powers, M. \& Vera-Zambrano, S. (2018). How journalists use social media in France and the United States: Analyzing technology use across journalistic fields. New Media \& Society, 20(8), 2728-2744. https://doi.org/10.1177/1461444817731566

Rodríguez-Ruibal, A. \& García-López, J. (2013). Uso y funciones de Twitter en periodistas españoles (Spanish Journalists: Use and Functions of Twitter). Estudios sobre el mensaje periodístico, 19, 963-969. https://doi.org/10.5209/rev_ESMP.2013.v19.42181

Rost, A. (2012). Periodismo e interactividad: preguntas, definiciones y desafíos en la participación de los usuarios (Journalism and interactivity: questions, definitions and challenges in user participation). In E. García-de-Torres (Coord.). Cartografía del periodismo participativo (Mapping of participatory journalism) (pp. 13-16). Tirant Lo Blanch.

Sánchez-Gonzales, H. \& Méndez-Muros, S. (2015). Las guías de uso de medios sociales: regularización periodística y calidad informativa (Usage guides of social media: journalistic regularization and informative quality). Estudios Sobre El Mensaje Periodístico, 21, 143-154. https://doi.org/10.5209/rev_ESMP.2015.v21.51136 
Santisteban G. \& Rodríguez, S. (2013). Twitter como herramienta para los medios tradicionales de comunicación en Colombia: su incidencia en el cambio de las prácticas periodísticas y la interacción con su público (Twitter as a tool for traditional communication media in Colombia: its impact on changing journalistic practices and interaction with its public) (Thesis, Universidad del Rosario, Argentina). Retrieved from http://repository.urosario.edu.co/handle/10336/4969

TandocJr, E.C., \&Vos, T.P. (2016). The journalist is marketing the news: Social media in the gatekeeping process. Journalism practice, 10(8), 950-966. https://doi.org/10.1080/17512786.2015.1087811

Ure, M. (2017). Engagement estratégico y encuentro conversacional en los medios sociales (Strategic engagement and conversational encounter in social media). Revista de Comunicación, 17(1), 181-196. https://doi.org/10.26441/RC17.1-2018-A10

\section{SOBRE LAS AUTORAS}

LILIANA GUTIÉRREZ-COBA, investigadora Senior (IS) Minciencias. Doctora en Ciencias de la Información por la Universidad del País Vasco (España), con énfasis en la investigación sobre temas relacionados con el periodismo, las tecnologías y la opinión pública. Es directora del Grupo de Investigación en Periodismo, GIP, de la Universidad de La Sabana (Colombia).

iD https://orcid.org/0000-0001-5532-8765

LAURA SILVA-GóMEZ, magister en Periodismo y Comunicación Digital por la Universidad de La Sabana (Colombia) e investigadora auxiliar en el Grupo de Investigación en Periodismo, GIP, de la misma institución. Es coordinadora de contenido en el portal web de Win Sports.

(iD) https://orcid.org/0000-0001-6672-9425 